\title{
Duality, reflexivity and atomic decompositions in Banach spaces
}

by

\author{
Daniel Carando and Silvia Lassalle (Buenos Aires)
}

\begin{abstract}
We study atomic decompositions and their relationship with duality and reflexivity of Banach spaces. To this end, we extend the concepts of "shrinking" and "boundedly complete" Schauder basis to the atomic decomposition framework. This allows us to answer a basic duality question: when an atomic decomposition for a Banach space generates, by duality, an atomic decomposition for its dual space. We also characterize the reflexivity of a Banach space in terms of properties of its atomic decompositions.
\end{abstract}

Introduction. The concept of Schauder basis for a Banach space $X$ is a natural way to introduce a coordinate system in the Banach space setting. This notion corresponds to orthogonal basis in Hilbert spaces. The biorthogonal dual sequence, in $X^{\prime}$, recovers the coordinates of the expansion of the elements of $X$ in terms of the basis. In general, this dual sequence is not necessarily a basis for the whole space $X^{\prime}$, but only for the closed subspace the sequence spans. In the context of nonharmonic Fourier series, Duffin and Schaeffer [9] introduced frames for Hilbert spaces. These are sequences that allow reconstruction formulae that need not be unique, as opposed to what happens with bases. This redundancy makes frames useful in different applications such as sampling theory and noise reduction in image and signal processing (see, for instance, $[1,7,16]$ ).

In the eighties, Gröchenig [12] introduced atomic decompositions as an extension of the notion of frames from Hilbert space theory to a Banach space setting. Another concept extending the notion of Hilbert space frames is that of Banach frames. Both new concepts have much in common and in many situations are equivalent. Loosely speaking, atomic decompositions involve the existence of a reconstruction formula as a series expansion, while Banach frames give rise to a reconstruction operator.

We concentrate on some mathematical aspects of atomic decompositions from a functional analytic point of view, within the framework of abstract

2000 Mathematics Subject Classification: Primary 41A65, 46B10; Secondary 42C15, 46B15.

Key words and phrases: atomic decompositions, Banach frames, duality, reflexivity. 
approximation theory in Banach spaces. The results obtained can be applied to the study of approximation of functions on Banach spaces admitting this type of structure (see, for example, [2]).

In this article, we relate duality and reflexivity with atomic decompositions. In the Schauder basis setting, properties of a basis of a Banach space $X$ allow us to determine if its dual basic sequence is actually a basis of $X^{\prime}$. Also, the reflexivity of $X$ can be characterized in terms of attributes of the basis $[13,14]$.

The study of duality for atomic decompositions has a natural approach. Indeed, if $\left(\left(x_{i}^{\prime}\right),\left(x_{i}\right)\right)$ is an atomic decomposition for $X$, we investigate conditions ensuring that $\left(\left(x_{i}\right),\left(x_{i}^{\prime}\right)\right)$ is an atomic decomposition for $X^{\prime}$. In order to deal with this question we introduce the concept of shrinking and strongly shrinking atomic decomposition, which extend the notion of shrinking Schauder basis. We show that an atomic decomposition for $X$ is shrinking if and only if the dual pair is an atomic decomposition for the dual space $X^{\prime}$. Examples are presented in order to clarify the situation and to compare it with the Schauder basis context.

A Banach space $X$ with Schauder basis is reflexive if and only if the basis is shrinking and boundedly complete. We introduce the notion of boundedly complete atomic decomposition and discuss the connection between these two properties of an atomic decomposition and the reflexivity of $X$.

For further information on atomic decompositions and Banach frames see, for example, $[5,6,12]$ and the references therein. We refer to $[8,15]$ for background in Banach space theory.

1. Duality for atomic decompositions. By a Banach sequence space we mean a Banach space of scalar sequences for which the coordinate functionals are continuous. We say that the space is a Schauder sequence space if, in addition, the unit vectors $\left\{e_{i}\right\}$ given by $\left(e_{i}\right)_{j}=\delta_{i, j}$ form a basis for it. In this case, a sequence $a=\left(a_{i}\right)$ can be written as $a=\sum_{i} a_{i} e_{i}$.

Definition 1.1. Let $X$ be a Banach space and $Z$ be a Banach sequence space. Let $\left(x_{i}^{\prime}\right)$ and $\left(x_{i}\right)$ be sequences in $X^{\prime}$ and $X$ respectively. We say that $\left(\left(x_{i}^{\prime}\right),\left(x_{i}\right)\right)$ is an atomic decomposition of $X$ with respect to $Z$ if for all $x \in X$ :

(a) $\left(\left\langle x_{i}^{\prime}, x\right\rangle\right) \in Z$,

(b) $A\|x\| \leq\left\|\left(\left\langle x_{i}^{\prime}, x\right\rangle\right)\right\|_{Z} \leq B\|x\|$, with $A$ and $B$ positive constants,

(c) $x=\sum_{i}\left\langle x_{i}^{\prime}, x\right\rangle x_{i}$.

We will often refer to property (c) in the above definition as the reconstruction formula associated to the atomic decomposition.

A separable Banach space admits an atomic decomposition if and only if it has the bounded approximation property (this result is contained in [17]). Moreover, if $\left(\left(x_{i}^{\prime}\right),\left(x_{i}\right)\right)$ is an atomic decomposition of $X$ with respect 
to some Banach sequence space $Z$, we can always find a Schauder sequence space $X_{d}$ and an operator $S: X_{d} \rightarrow X$ such that $S e_{i}=x_{i}$ and $\left(\left(x_{i}^{\prime}\right),\left(x_{i}\right)\right)$ is also an atomic decomposition of $X$ with respect to $X_{d}[17,4]$. In the following, we consider atomic decompositions of the form $\left(\left(x_{i}^{\prime}\right),\left(S e_{i}\right)\right)$ associated to a Schauder sequence space $X_{d}$. See [3] for a complete treatment of the approximation property.

Whenever a sequence $\left(x_{i}^{\prime}\right) \subset X^{\prime}$ satisfies conditions (a) and (b) of Definition 1.1 and there exists a linear operator $S: X_{d} \rightarrow X$ satisfying $S\left(\left\langle x_{i}^{\prime}, x\right\rangle\right)=x$ for all $x \in X$, the pair $\left(\left(x_{i}^{\prime}\right), S\right)$ is said to be a Banach frame for $X$ with respect to $X_{d}[4,5]$. Note that any atomic decomposition associated to an operator $S$ defines a Banach frame, with the additional property of the reconstruction formula given by property (c) (which is stronger than having the identity $\left.S\left(\left\langle x_{i}^{\prime}, x\right\rangle\right)=x\right)$. In the case where $X_{d}$ is a Schauder sequence space, $\left(\left(x_{i}^{\prime}\right),\left(S e_{i}\right)\right)$ is an atomic decomposition if and only if $\left(\left(x_{i}^{\prime}\right), S\right)$ is a Banach frame. Therefore, all the results stated below for atomic decompositions can be reformulated for Banach frames with respect to Schauder sequence spaces.

If $\left(\left(x_{i}^{\prime}\right),\left(S e_{i}\right)\right)$ is an atomic decomposition of $X$ with respect to $X_{d}$, we define the natural inclusion $J: X \rightarrow X_{d}$ by

$$
J(x)=\left(\left\langle x_{i}^{\prime}, x\right\rangle\right)=\sum_{i}\left\langle x_{i}^{\prime}, x\right\rangle e_{i} .
$$

If $\left(e_{i}^{\prime}\right)$ is the dual basic sequence of $\left(e_{i}\right)$, then $x_{i}^{\prime}=J^{\prime} e_{i}^{\prime}$. Note that $S J=I_{X}$ and so $X$ is isomorphic to a complemented subspace of $X_{d}$. Conversely, we have the following

REMARK 1.2. Let $X$ be a Banach space and $X_{d}$ be a Schauder sequence space. Suppose there exist continuous operators $J: X \rightarrow X_{d}$ and $S: X_{d} \rightarrow X$ such that $S J=I_{X}$. Then, if $\left(e_{i}^{\prime}\right)$ is the dual basic sequence of $\left(e_{i}\right)$, the pair $\left(\left(J^{\prime} e_{i}^{\prime}\right),\left(S e_{i}\right)\right)$ is an atomic decomposition for $X$ with respect to $X_{d}$.

Indeed, condition (a) in the definition is satisfied since $J x$ belongs to $X_{d}$ for all $x \in X$. Condition (c) holds by the continuity of $S$, and the constants $A$ and $B$ of condition (b) may be chosen to be $\|S\|^{-1}$ and $\|J\|$ respectively.

A natural question arises: if $\left(\left(x_{i}^{\prime}\right),\left(S e_{i}\right)\right)$ is an atomic decomposition for $X$, is $\left(\left(S e_{i}\right),\left(x_{i}^{\prime}\right)\right)$ an atomic decomposition for $X^{\prime}$ ?

The notion of shrinking basis gives an answer to this question in the case of Schauder bases instead of atomic decompositions. Indeed, for a Schauder basis $\left(x_{i}\right)$ of $X$, its dual sequence $\left(x_{i}^{\prime}\right)$ is a Schauder basis of $X^{\prime}$ if and only if $\left(x_{i}\right)$ is shrinking. Recall that $\left(x_{i}\right)$ is said to be shrinking if for all $x^{\prime} \in X^{\prime}$,

$$
\lim _{N \rightarrow \infty}\left\|\left.x^{\prime}\right|_{\left[x_{i}: i \geq N\right]}\right\|=0
$$

where $\left[x_{i}: i \geq N\right]$ is the subspace spanned by $\left\{x_{i}\right\}_{i \geq N}$. 
Our goal is then to extend the notion of shrinking basis to shrinking atomic decompositions. A proper definition for a shrinking atomic decomposition should satisfy the following: an atomic decomposition $\left(\left(x_{i}^{\prime}\right),\left(S e_{i}\right)\right)$ of $X$ with respect to $X_{d}$ is shrinking if and only if $\left(\left(S e_{i}\right),\left(x_{i}^{\prime}\right)\right)$ is an atomic decomposition of $X^{\prime}$ with respect to some sequence space. In order to establish the correct definition, we believe the following discussion is necessary.

First, note that if $X_{d}$ is a Schauder sequence space, then the dual $\left(X_{d}\right)^{\prime}$ is a Banach sequence space, provided we identify each linear functional $b^{\prime} \in$ $\left(X_{d}\right)^{\prime}$ with the sequence $\left(\left\langle b^{\prime}, e_{i}\right\rangle\right)$.

One way to ensure that $\left(\left(S e_{i}\right),\left(x_{i}^{\prime}\right)\right)$ is an atomic decomposition of $X^{\prime}$ is to require that $\left(e_{i}\right)$ be a shrinking basis of $X_{d}$ (see Proposition 1.9). In this case, $\left(e_{i}^{\prime}\right)$ turns out to be a basis of $\left(X_{d}\right)^{\prime}$ and this last space is therefore a Schauder sequence space.

Another natural attempt is to impose that

$$
\lim _{N \rightarrow \infty}\left\|\left.x^{\prime}\right|_{\left[S e_{i}: i \geq N\right]}\right\|=0 \quad \text { for all } x^{\prime} \in X^{\prime} .
$$

As we will see, condition (1.2) implies that $\left(\left(S e_{i}\right),\left(x_{i}^{\prime}\right)\right)$ is an atomic decomposition of $X^{\prime}$. This condition is the most direct extension of a shrinking basis. However, it does not take into account the linear functionals $\left(x_{i}^{\prime}\right)$. Although for a basis the dual linear functionals are uniquely determined, the sequence $\left(S e_{i}\right)$ by itself does not characterize the atomic decomposition. Therefore, one should not expect (1.2) to be the appropriate definition for a shrinking atomic decomposition.

In fact, none of the previous two conditions is equivalent to $\left(\left(S e_{i}\right),\left(x_{i}^{\prime}\right)\right)$ being an atomic decomposition of $X^{\prime}$. Moreover, we will see that both conditions are strictly stronger than desired (see Proposition 1.9 and Examples 1.10, 1.11).

Although we state our results for atomic decompositions which are equivalent to Banach frames, we want to give the definition of shrinking atomic decompositions for the general case. Let, then, $\left(\left(x_{i}^{\prime}\right),\left(x_{i}\right)\right)$ be an atomic decomposition of $X$ with respect to a Banach sequence space $Z$. For each $N \in \mathbb{N}$, define the linear operator $T_{N}: X \rightarrow X$ by

$$
T_{N}(x)=\sum_{i \geq N}\left\langle x_{i}^{\prime}, x\right\rangle x_{i} .
$$

Each $T_{N}$ is a bounded linear operator as a consequence of the BanachSteinhaus theorem. Also, again by that theorem, they are uniformly bounded. Now we define:

Definition 1.3. Let $X$ be a Banach space, $Z$ be a Banach sequence space and $\left(\left(x_{i}^{\prime}\right),\left(x_{i}\right)\right)$ be an atomic decomposition of $X$ with respect to $Z$. 
We say that $\left(\left(x_{i}^{\prime}\right),\left(x_{i}\right)\right)$ is shrinking if for all $x^{\prime} \in X^{\prime}$,

$$
\left\|x^{\prime} \circ T_{N}\right\| \underset{N \rightarrow \infty}{\longrightarrow} 0,
$$

where $T_{N}$ is defined by (1.3).

Theorem 1.4. Let $X$ be a Banach space and $X_{d}$ be a Schauder sequence space. Let $S: X_{d} \rightarrow X$ be a continuous operator and $\left(x_{i}^{\prime}\right) \subset X^{\prime}$ be a sequence so that $\left(\left(x_{i}^{\prime}\right),\left(S e_{i}\right)\right)$ is an atomic decomposition for $X$ with respect to $X_{d}$. Then $\left(\left(S e_{i}\right),\left(x_{i}^{\prime}\right)\right)$ is an atomic decomposition for $X^{\prime}$ with respect to $\left(X_{d}\right)^{\prime}$ if and only if $\left(\left(x_{i}^{\prime}\right),\left(S e_{i}\right)\right)$ is shrinking.

Proof. Assume that $\left(\left(x_{i}^{\prime}\right),\left(S e_{i}\right)\right)$ is a shrinking atomic decomposition for $X$ with respect to $X_{d}$. Since $\left\langle x^{\prime}, S e_{i}\right\rangle=\left\langle S^{\prime} x^{\prime}, e_{i}\right\rangle$ for all $i$, the sequence $\left(\left\langle x^{\prime}, S e_{i}\right\rangle\right)$ belongs to $\left(X_{d}\right)^{\prime}$ considered as a Banach sequence space, and condition (a) is satisfied.

To show that the norms $\left\|x^{\prime}\right\|$ and $\left\|\left(\left\langle x^{\prime}, S e_{i}\right\rangle\right)\right\|$ are equivalent, note that $S$ is surjective, therefore $S^{\prime}$ is an isomorphism onto its image.

Finally, we have to show the validity of the reconstruction formula, i.e., $x^{\prime}=\sum_{i}\left\langle x^{\prime}, x_{i}\right\rangle x_{i}^{\prime}$, where $x_{i}=S e_{i}$. It is clear that $\left\langle x^{\prime}, x\right\rangle=\sum_{i}\left\langle x^{\prime}, x_{i}\right\rangle\left\langle x_{i}^{\prime}, x\right\rangle$ for all $x \in X$, so it only remains to show that the series $\sum_{i}\left\langle x^{\prime}, x_{i}\right\rangle x_{i}^{\prime}$ converges. Let us see that it is a Cauchy series:

$$
\begin{aligned}
\sup _{\|x\| \leq 1}\left|\sum_{i=N}^{M-1}\left\langle x^{\prime}, x_{i}\right\rangle\left\langle x_{i}^{\prime}, x\right\rangle\right| & =\sup _{\|x\| \leq 1}\left|\left\langle x^{\prime},\left(T_{M}-T_{N}\right) x\right\rangle\right| \\
& \leq\left\|x^{\prime} \circ T_{M}\right\|+\left\|x^{\prime} \circ T_{N}\right\| \underset{N, M \rightarrow \infty}{\longrightarrow} 0 .
\end{aligned}
$$

Therefore, we have shown that $\left(\left(S e_{i}\right),\left(x_{i}^{\prime}\right)\right)$ is an atomic decomposition for $X^{\prime}$ with respect to $\left(X_{d}\right)^{\prime}$.

The converse follows immediately from

$$
\left\|x^{\prime} \circ T_{N}\right\|=\sup _{\|x\| \leq 1}\left|\left\langle x^{\prime}, T_{N} x\right\rangle\right|=\left\|\sum_{i \geq N}\left\langle x^{\prime}, S e_{i}\right\rangle x_{i}^{\prime}\right\|
$$

and the fact that $\left(\left(S e_{i}\right),\left(x_{i}^{\prime}\right)\right)$ is an atomic decomposition for $X^{\prime}$ with respect to $\left(X_{d}\right)^{\prime}$.

If $X_{d}$ is a Schauder sequence space, it is easy to see that the pair $\left(\left(S e_{i}\right), J^{\prime}\right)$ defines a Banach frame for $X^{\prime}$ with respect to $\left(X_{d}\right)^{\prime}$, even if the atomic decomposition $\left(\left(x_{i}^{\prime}\right),\left(S e_{i}\right)\right)$ is not shrinking. Indeed, since $J^{\prime} S^{\prime}=\operatorname{Id}_{X^{\prime}}$ and $S^{\prime} x^{\prime}=\left(\left\langle x^{\prime}, S e_{i}\right\rangle\right)_{i}$, we have $J^{\prime}\left(\left\langle x^{\prime}, S e_{i}\right\rangle\right)=x^{\prime}$. The continuity of $S$ and $J$ gives the frame bounds. However, in this case the reconstruction formula does not necessarily hold.

On the other hand, if $\left(\left(x_{i}^{\prime}\right),\left(S e_{i}\right)\right)$ is shrinking and the range of $S^{\prime}$ is contained in the closed subspace of $\left(X_{d}\right)^{\prime}$ spanned by the dual sequence $\left(e_{i}^{\prime}\right)$, then 
we can obtain an atomic decomposition for $X^{\prime}$ with respect to a Schauder sequence space. This fact motivates the definition of strongly shrinking atomic decompositions. First we state the following corollary, which is an immediate consequence of the relationship between atomic decompositions and the bounded approximation property:

COROllary 1.5. Suppose that $X$ admits a shrinking atomic decomposition $\left(\left(x_{i}^{\prime}\right),\left(S e_{i}\right)\right)$. Then $X^{\prime}$ is separable and has the bounded approximation property.

The existence of the operator $S$ provides us with a sequence $\left(S_{N}\right)$ of continuous operators which is useful to avoid the obstacle arising from the fact that we work with nonunique representations.

Fix $N$, and consider the operator $S_{N}: X_{d} \rightarrow X$ defined by

$$
S_{N}(a)=\sum_{i \geq N} a_{i} x_{i}
$$

Then $\left(S_{N}\right)$ is bounded by $2 K\|S\|$, where $K$ is the basis constant of $\left(e_{i}\right)$.

Definition 1.6. Let $X$ be a Banach space, $X_{d}$ be a Schauder sequence space, and $S: X_{d} \rightarrow X$ be a continuous operator such that $\left(\left(x_{i}^{\prime}\right),\left(S e_{i}\right)\right)$ is an atomic decomposition of $X$ with respect to $X_{d}$. We say that $\left(\left(x_{i}^{\prime}\right),\left(S e_{i}\right)\right)$ is strongly shrinking if for all $x^{\prime} \in X^{\prime}$,

$$
\left\|x^{\prime} \circ S_{N}\right\| \rightarrow 0 \quad \text { as } N \rightarrow \infty,
$$

where $S_{N}: X_{d} \rightarrow X$ is given by $S_{N}(a)=\sum_{i \geq N} a_{i} x_{i}$.

It is clear that any strongly shrinking atomic decomposition is shrinking, since $T_{N}=S_{N} \circ J$. On the other hand, at the end of this section we present examples showing that the converse is not true.

Before stating the duality property of strongly shrinking atomic decompositions we need the following:

Lemma 1.7. Let $X$ be a Banach space and $X_{d}$ be a Schauder sequence space. Let $S: X_{d} \rightarrow X$ be a continuous operator. If $\left(\left(x_{i}^{\prime}\right),\left(S e_{i}\right)\right)$ is a strongly shrinking atomic decomposition then $S^{\prime}\left(X^{\prime}\right)$ is contained in the closure of $\left[e_{i}^{\prime}: i \geq 1\right]$ in $\left(X_{d}\right)^{\prime}$ and $S^{\prime} x^{\prime}=\sum_{i}\left\langle x^{\prime}, S e_{i}\right\rangle e_{i}^{\prime}$ for all $x^{\prime} \in X^{\prime}$.

Proof. Put $x_{i}=S e_{i}$. Since $\left\langle S^{\prime} x^{\prime}, e_{i}\right\rangle=\left\langle x^{\prime}, x_{i}\right\rangle$, it is enough to see that $\sum_{i=1}^{M}\left\langle x^{\prime}, x_{i}\right\rangle e_{i}^{\prime}$ is a Cauchy sequence in $\left(X_{d}\right)^{\prime}$. Now

$$
\begin{aligned}
\left\|\sum_{i=N}^{M-1}\left\langle x^{\prime}, x_{i}\right\rangle e_{i}^{\prime}\right\| & =\sup _{\|a\| \leq 1}\left|\sum_{i=N}^{M-1}\left\langle x^{\prime}, x_{i}\right\rangle\left\langle e_{i}^{\prime}, a\right\rangle\right|=\sup _{\|a\| \leq 1}\left|\left\langle x^{\prime}, \sum_{i=N}^{M-1} a_{i} x_{i}\right\rangle\right| \\
& =\sup _{\|a\| \leq 1}\left|\left\langle x^{\prime},\left(S_{N}-S_{M}\right) a\right\rangle\right| \leq\left\|x^{\prime} \circ S_{N}\right\|+\left\|x^{\prime} \circ S_{M}\right\| .
\end{aligned}
$$

Since $\left(\left(x_{i}^{\prime}\right),\left(S e_{i}\right)\right)$ is strongly shrinking, the result follows. 
If $X$ is a Banach space and $X_{d}$ is an associated Schauder sequence space with basis $\left(e_{i}\right)$, we denote by $X_{d}^{\prime}$ the closed subspace spanned by $\left(e_{i}^{\prime}\right)$ in $\left(X_{d}\right)^{\prime}$, where $\left(e_{i}^{\prime}\right)$ is the dual sequence of $\left(e_{i}\right)$. Since $\left(e_{i}^{\prime}\right)$ is a basic sequence, $X_{d}^{\prime}$ is a Schauder sequence space.

Now we can establish:

TheOREM 1.8. Let $X$ be a Banach space and $X_{d}$ be a Schauder sequence space. Let $S: X_{d} \rightarrow X$ be a continuous operator and $\left(x_{i}^{\prime}\right) \subset X^{\prime}$ be a sequence such that $\left(\left(x_{i}^{\prime}\right),\left(S e_{i}\right)\right)$ is an atomic decomposition for $X$ with respect to $X_{d}$. Then $\left(\left(S e_{i}\right),\left(x_{i}^{\prime}\right)\right)$ is an atomic decomposition for $X^{\prime}$ with respect to $X_{d}^{\prime}$ if and only if $\left(\left(x_{i}^{\prime}\right),\left(S e_{i}\right)\right)$ is strongly shrinking.

Proof. If $\left(\left(x_{i}^{\prime}\right),\left(S e_{i}\right)\right)$ is a strongly shrinking atomic decomposition for $X$ with respect to $X_{d}$, it is shrinking. Then a combination of Lemma 1.7 and Theorem 1.4 shows that $\left(\left(S e_{i}\right),\left(x_{i}^{\prime}\right)\right)$ is an atomic decomposition for $X^{\prime}$ with respect to $X_{d}^{\prime}$. The converse follows as in the proof of Theorem 1.4.

Now we see that our first two candidates for the definition of shrinking atomic decomposition are strictly stronger than the proper definition. Moreover, they are even stronger than the definition of strongly shrinking atomic decomposition. First we have

Proposition 1.9. Let $X$ be a Banach space, $X_{d}$ be a sequence space and $S: X_{d} \rightarrow X$ be a continuous operator such that $\left(\left(x_{i}^{\prime}\right),\left(S e_{i}\right)\right)$ is an atomic decomposition of $X$ with respect to $X_{d}$. Consider the following statements:

(a) the basis $\left(e_{i}\right)$ of $X_{d}$ is shrinking,

(b) $\left(S e_{i}\right)$ satisfies condition $(1.2)$, i.e. $\lim _{N \rightarrow \infty}\left\|\left.x^{\prime}\right|_{\left[S e_{i}: i \geq N\right]}\right\|=0$ for all $x^{\prime} \in X^{\prime}$.

Then either (a) or (b) implies that $\left(\left(x_{i}^{\prime}\right),\left(S e_{i}\right)\right)$ is a strongly shrinking atomic decomposition.

Proof. If (a) holds, let $K$ be the basis constant of the shrinking basis $\left(e_{i}\right)$ and $F_{N}=\left[e_{i}: i \geq N\right]$. We have

$$
\begin{aligned}
\left\|x^{\prime} \circ S_{N}\right\| & =\sup _{\|a\| \leq 1}\left|\left\langle S^{\prime} x^{\prime}, \sum_{i \geq N} a_{i} e_{i}\right\rangle\right| \leq \sup _{\substack{a \in F_{N} \\
\|a\| \leq 1+K}}\left|\left\langle S^{\prime} x^{\prime}, \sum_{i \geq N} a_{i} e_{i}\right\rangle\right| \\
& =(1+K)\left\|\left.S^{\prime} x^{\prime}\right|_{F_{N}}\right\|,
\end{aligned}
$$

which goes to zero since $\left(e_{i}\right)$ is shrinking. The proof for (b) is straightforward.

Now, we present counterexamples for the converses.

EXAMPLE 1.10 (A strongly shrinking atomic decomposition for which statement (a) of Proposition 1.9 does not hold). Take $X=\ell_{2}, X_{d}=\ell_{1} \oplus \ell_{2}$ with the basis $\left(f_{k}\right)$ that alternates $\left(e_{i}, 0\right)$ and $\left(0, e_{i}\right)$. Consider the projection 
$S: X_{d} \rightarrow X$ on the second coordinate. If we define $x_{2 j-1}^{\prime}=0$ and $x_{2 j}^{\prime}=e_{j}^{\prime}$ on $X$ for $j=1,2, \ldots$, then $\left(\left(x_{k}^{\prime}\right),\left(S f_{k}\right)\right)$ is a strongly shrinking atomic decomposition of $X$ with respect to $X_{d}$, since condition (b) of Proposition 1.9 is satisfied. However, the basis for $X_{d}$ is not shrinking. Indeed, $a^{\prime}=$ $(1,1, \ldots) \times(0,0, \ldots) \in \ell_{\infty} \times \ell_{2}$ satisfies $\left\|\left.a^{\prime}\right|_{\left[f_{k}: k \geq N\right]}\right\|=1$ for all $N$.

EXAMPLE 1.11 (A strongly shrinking atomic decomposition for which statement (b) of Proposition 1.9 does not hold). We show a family of examples of strongly shrinking atomic decompositions not satisfying condition (b). Take $X=X_{d}=\ell_{2}$, fix $b \in \ell_{2}$ with $b_{j} \neq 0$ for all $j$ and $b_{1}=1$ and consider the operator $S: X_{d} \rightarrow X$ given by the infinite matrix

$$
\left(\begin{array}{ccccccccccccccccc}
b_{1} & b_{2} & 0 & b_{3} & 0 & 0 & b_{4} & 0 & 0 & 0 & b_{5} & 0 & 0 & 0 & 0 & b_{6} & \cdots \\
0 & 0 & b_{1} & 0 & b_{2} & 0 & 0 & b_{3} & 0 & 0 & 0 & b_{4} & 0 & 0 & 0 & 0 & \cdots \\
0 & 0 & 0 & 0 & 0 & b_{1} & 0 & 0 & b_{2} & 0 & 0 & 0 & b_{3} & 0 & 0 & 0 & \cdots \\
0 & 0 & 0 & 0 & 0 & 0 & 0 & 0 & 0 & b_{1} & 0 & 0 & 0 & b_{2} & 0 & 0 & \cdots \\
\vdots & \vdots & \vdots & & & & & & & & & & & & & & \ddots
\end{array}\right)
$$

Note that each column of the matrix has a unique nonzero coefficient. Then consider the subsequences

$$
N_{i}=\{j \in \mathbb{N} \text { : the element in column } j \text { and row } i \text { is nonzero }\}
$$

obtaining the disjoint partition $\mathbb{N}=\bigcup_{i} N_{i}$. If we write $N_{i}=\left\{\left(n_{i j}\right)_{j}\right\}$, for any $a \in \ell_{2}$ we obtain

$$
S a=\sum_{i=1}^{\infty}\left(\sum_{j=1}^{\infty} b_{j} a_{n_{i j}}\right) e_{i} .
$$

Also, note that for each $i$, the element in row $i$ and column $i(i+1) / 2$ is $b_{1}=1$. This means that $S e_{i(i+1) / 2}=e_{i}$ for all $i$.

Hölder's inequality gives the continuity of $S$ :

$$
\|S a\|^{2}=\sum_{i=1}^{\infty}\left|\sum_{j=1}^{\infty} b_{j} a_{n_{i j}}\right|^{2} \leq \sum_{i=1}^{\infty}\left(\sum_{j=1}^{\infty}\left|b_{j}\right|^{2}\right)\left(\sum_{j=1}^{\infty}\left|a_{n_{i j}}\right|^{2}\right)=\|b\|^{2}\|a\|^{2} .
$$

Let $x_{k}^{\prime}=e_{i}^{\prime}$ if $k=i(i+1) / 2$ and $x_{k}^{\prime}=0$ otherwise. It is clear that $\left(\left\langle x_{k}^{\prime}, x\right\rangle\right)_{k} \in X_{d}$ for all $x \in \ell_{2}$. Also,

$$
\sum_{k=1}^{\infty}\left\langle x_{k}^{\prime}, x\right\rangle S e_{k}=\sum_{i=1}^{\infty}\left\langle x_{i(i+1) / 2}^{\prime}, x\right\rangle S e_{i(i+1) / 2}=\sum_{i=1}^{\infty}\left\langle e_{i}^{\prime}, x\right\rangle e_{i}=x .
$$

If $J: X \rightarrow X_{d}$ is the canonical inclusion, $\|J x\| \leq\|x\|$ and $\|x\|=\|S J x\| \leq$ $\|b\|\left\|\left(\left\langle x_{i}^{\prime}, x\right\rangle\right)\right\|$, and then $\left(\left(x_{i}^{\prime}\right),\left(S e_{i}\right)\right)$ is an atomic decomposition.

To see it is strongly shrinking, it is enough to observe that condition (a) of Proposition 1.9 holds. On the other hand, for any $N$, the subspace 
$\left[S e_{i}: i \geq N\right]$ is in fact all $\ell_{2}$. Therefore, condition (b) of Proposition 1.9 is not satisfied.

It is clear that different examples can be obtained for any disjoint partition $\mathbb{N}=\bigcup_{i} N_{i}$.

Note that in Example 1.10 the atomic decomposition satisfies condition (b) but not (a) while in Example 1.11 condition (a) is satisfied but (b) is not. A direct combination of both examples provides a strongly shrinking atomic decomposition for which neither (a) nor (b) of Remark 1.9 is satisfied.

The following examples show that shrinking and strongly shrinking are not equivalent notions for atomic decompositions. Note that these two concepts coincide for Schauder bases. Also, a Schauder basis of a reflexive Banach space is always shrinking, and consequently strongly shrinking. The examples show that a reflexive space can admit an atomic decomposition that is not strongly shrinking. The first example is a modification of Example 1.10. We thank the referee for kindly suggesting it to us.

EXAMPLE 1.12 (An atomic decomposition for a reflexive Banach space $X$ which is shrinking but not strongly shrinking). Take $X=\ell_{2}, X_{d}=\ell_{1} \oplus \ell_{2}$ with the basis $\left(f_{k}\right)$ that alternates $\left(e_{i}, 0\right)$ and $\left(0, e_{i}\right)$. We define $S: X_{d} \rightarrow X$ by $S\left(e_{i}, 0\right)=e_{1}, S\left(0, e_{i}\right)=e_{i}$, and $x_{2 j-1}^{\prime}=0$ and $x_{2 j}^{\prime}=e_{j}^{\prime}$ on $X$ for $j=1,2, \ldots$. Since $\left\|\left(e_{1}^{\prime}, 0\right) \circ S_{N}\right\|=1$ for all $N$, we see that $\left(\left(x_{k}^{\prime}\right),\left(S f_{k}\right)\right)$ is not strongly shrinking. By a direct calculation or using Theorem 1.4 we see that it is shrinking.

EXAMPLE 1.13 (Another atomic decomposition for a reflexive Banach space $X$ which is shrinking but not strongly shrinking). Let $X=\ell_{2}$ and let $X_{d}$ be the full 3-fold projective tensor product $X_{d}=\bigotimes_{\pi}^{3} \ell_{2}$. This is a sequence space with basis $\left(e_{\alpha}\right)_{\alpha \in \mathbb{N}^{3}}$, where $e_{\alpha}=e_{\alpha_{1}} \otimes e_{\alpha_{2}} \otimes e_{\alpha_{3}}$ and $\mathbb{N}^{3}$ is considered with the square ordering [10] (see also [11]). Let $\Phi$ be the bilinear form on $\ell_{2} \times \ell_{2}$ given by $\Phi(y, z)=\sum_{k \geq 1} y_{k} z_{k}$. Consider the continuous operators $S: X_{d} \rightarrow X, S(x \otimes y \otimes z)=\Phi(y, z) x$, and $J: X \rightarrow X_{d}, J(x)=x \otimes$ $e_{1} \otimes e_{1}$, and set $x_{\alpha}^{\prime}=J^{\prime}\left(e_{\alpha_{1}}^{\prime} \otimes e_{\alpha_{2}}^{\prime} \otimes e_{\alpha_{3}}^{\prime}\right)$. Note that $S\left(e_{\alpha_{1}} \otimes e_{\alpha_{2}} \otimes e_{\alpha_{3}}\right)=e_{\alpha_{1}}$. We claim that the pair $\left(\left(x_{\alpha}^{\prime}\right)_{\alpha \in \mathbb{N}^{3}},\left(e_{\alpha_{1}}\right)_{\alpha \in \mathbb{N}^{3}}\right)$ is a shrinking atomic decomposition for $\ell_{2}$ with respect to $\bigotimes_{\pi}^{3} \ell_{2}$ which is not strongly shrinking. By Remark 1.2, to see that it is an atomic decomposition we only need to show that $S J=I_{\ell_{2}}$, which is clear since $\Phi\left(e_{1}, e_{1}\right)=1$.

To see that $\left(\left(x_{\alpha}^{\prime}\right),\left(e_{\alpha_{1}}\right)\right)$ is shrinking, by Theorem 1.4 , it is enough to check the reconstruction formula for each $x^{\prime} \in\left(\ell_{2}\right)^{\prime}=\ell_{2}$ with respect to the dual atomic decomposition $\left(\left(e_{\alpha_{1}}\right),\left(x_{\alpha}^{\prime}\right)\right)$. Since $x_{\alpha}^{\prime}=\delta_{\alpha_{2}, 1} \cdot \delta_{\alpha_{3}, 1} \cdot e_{\alpha_{1}}^{\prime}$, we have

$$
x^{\prime}=\sum_{\alpha_{1} \in \mathbb{N}}\left\langle x^{\prime}, e_{\alpha_{1}}\right\rangle e_{\alpha_{1}}^{\prime}=\sum_{\alpha \in \mathbb{N}^{3}}\left\langle x^{\prime}, e_{\alpha_{1}}\right\rangle \delta_{\alpha_{2}, 1} \cdot \delta_{\alpha_{3}, 1} \cdot e_{\alpha_{1}}^{\prime}=\sum_{\alpha \in \mathbb{N}^{3}}\left\langle x^{\prime}, e_{\alpha_{1}}\right\rangle x_{\alpha}^{\prime} .
$$


Now, suppose it is a strongly shrinking atomic decomposition. By Lemma 1.7, $S^{\prime}\left(X^{\prime}\right)$ should be contained in $X_{d}^{\prime}=\overline{\left[\left(e_{\alpha}\right)^{\prime}\right]}$. Identifying $\left(\bigotimes_{\pi}^{3} \ell_{2}\right)^{\prime}$ with the space of 3 -linear forms on $\ell_{2}$, we conclude that $S^{\prime}\left(X^{\prime}\right)$ must consist of trilinear forms which are separately weakly continuous on bounded sets.

Given $x^{\prime} \in X^{\prime}$ choose a unit vector $x \in \ell_{2}$ so that $\left\langle x^{\prime}, x\right\rangle=\left\|x^{\prime}\right\|$. Then, as a trilinear form,

$$
S^{\prime}\left(x^{\prime}\right)(x, y, z)=\left\langle x^{\prime}, S(x \otimes y \otimes z)\right\rangle=\Phi(y, z)\left\langle x^{\prime}, x\right\rangle .
$$

Now, $\left(e_{N}\right)_{N}$ is a weakly null sequence in $\ell_{2}$ but $S^{\prime}\left(x^{\prime}\right)\left(x, e_{N}, e_{N}\right)=\left\|x^{\prime}\right\|$. Therefore, $S^{\prime}\left(x^{\prime}\right)$ does not belong to $X_{d}^{\prime}$ for any nonzero $x^{\prime} \in X^{\prime}$ and the atomic decomposition is not strongly shrinking.

2. Atomic decompositions and reflexivity. Banach spaces with Schauder basis admit a characterization of reflexivity in terms of the properties of the basis. To be precise, a Banach space $X$ with Schauder basis $\left(e_{i}\right)$ is reflexive if and only if $\left(e_{i}\right)$ is shrinking and boundedly complete.

Recall that a Schauder basis $\left(e_{i}\right)$ of $X$ is said to be boundedly complete if for each scalar sequence $\left(a_{i}\right)$ such that

$$
\sup _{N}\left\|\sum_{i=1}^{N} a_{i} e_{i}\right\|<\infty
$$

the series $\sum_{i=1}^{\infty} a_{i} e_{i}$ converges in $X$. The following proposition establishes an equivalent formulation of this definition. This result can be found, for instance, in [18, Theorem II.6.2]. Nevertheless, we prefer to include a proof for the sake of completeness.

Proposition 2.1. Let $X$ be a Schauder sequence space with basis $\left(e_{i}\right)$. Then $\left(e_{i}\right)$ is boundedly complete if and only if for all $x^{\prime \prime} \in X^{\prime \prime}$, the series $\sum_{i=1}^{\infty}\left\langle x^{\prime \prime}, e_{i}^{\prime}\right\rangle e_{i}$ converges.

Proof. Suppose $\left(e_{i}\right)$ is boundedly complete with basis constant $K$. Take $x^{\prime \prime} \in X^{\prime \prime}$. To show that $\sum_{i=1}^{N}\left\langle x^{\prime \prime}, e_{i}^{\prime}\right\rangle e_{i}$ is uniformly bounded we consider its norm as the supremum over $x^{\prime} \in B_{X^{\prime}}$; then

$$
\left|\left\langle x^{\prime}, \sum_{i=1}^{N}\left\langle x^{\prime \prime}, e_{i}^{\prime}\right\rangle e_{i}\right\rangle\right|=\left|\left\langle x^{\prime \prime}, \sum_{i=1}^{N}\left\langle x^{\prime}, e_{i}\right\rangle e_{i}^{\prime}\right\rangle\right| \leq\left\|x^{\prime \prime}\right\|\left\|\sum_{i=1}^{N}\left\langle x^{\prime}, e_{i}\right\rangle e_{i}^{\prime}\right\| .
$$

Since

$$
\left\|\sum_{i=1}^{N}\left\langle x^{\prime}, e_{i}\right\rangle e_{i}^{\prime}\right\|=\sup _{\|x\| \leq 1}\left|\left\langle x^{\prime}, \sum_{i=1}^{N}\left\langle e_{i}^{\prime}, x\right\rangle e_{i}\right\rangle\right| \leq K\left\|x^{\prime}\right\|,
$$

we see that $K\left\|x^{\prime \prime}\right\|$ is a bound for $\left\|\sum_{i=1}^{N}\left\langle x^{\prime \prime}, e_{i}^{\prime}\right\rangle e_{i}\right\|$, for all $N \in \mathbb{N}$.

For the converse, take a scalar sequence $\left(a_{i}\right)$ such that $\sup _{N}\left\|\sum_{i=1}^{N} a_{i} e_{i}\right\|$ is bounded and consider $\varphi_{N}=\sum_{i=1}^{N} a_{i} e_{i} \in X^{\prime \prime}$. Then there exists a subnet 
$\left(\varphi_{n(\alpha)}\right)$ of $\left(\varphi_{N}\right)$ and $x^{\prime \prime} \in X^{\prime \prime}$ so that $x^{\prime \prime}=w^{*}-\lim \varphi_{n(\alpha)}$. Then $\left\langle x^{\prime \prime}, e_{k}^{\prime}\right\rangle=a_{k}$ for each $k \in \mathbb{N}$, and therefore $\sum_{i=1}^{\infty} a_{i} e_{i}=\sum_{i=1}^{\infty}\left\langle x^{\prime \prime}, e_{i}^{\prime}\right\rangle e_{i}$ converges.

Note that the equivalent condition involves both the basis $\left(e_{i}\right)_{i}$ and its dual basic sequence $\left(e_{i}^{\prime}\right)_{i}$. Therefore, this formulation is more suitable for extension to atomic decompositions:

Definition 2.2. Let $X$ be a Banach space and let $Z$ be a Banach sequence space. Let $\left(\left(x_{i}^{\prime}\right),\left(x_{i}\right)\right)$ be an atomic decomposition of $X$ with respect to $Z$. The atomic decomposition is said to be boundedly complete if for each $x^{\prime \prime} \in X^{\prime \prime}$, the series $\sum_{i=1}^{\infty}\left\langle x^{\prime \prime}, x_{i}^{\prime}\right\rangle x_{i}$ converges in $X$.

It is immediate that whenever $X$ is reflexive, any atomic decomposition is boundedly complete. Moreover, we have:

Proposition 2.3. Let $X$ be a Banach space and $X_{d}$ be a Schauder sequence space. Let $S: X_{d} \rightarrow X$ be a continuous operator and $\left(x_{i}^{\prime}\right) \subset X^{\prime}$ be a sequence such that $\left(\left(x_{i}^{\prime}\right),\left(S e_{i}\right)\right)$ is a shrinking atomic decomposition for $X$ with respect to $X_{d}$. Then $\left(\left(S e_{i}\right),\left(x_{i}^{\prime}\right)\right)$ is a boundedly complete atomic decomposition for $X^{\prime}$ with respect to $\left(X_{d}\right)^{\prime}$.

Proof. Let $\varrho: X^{\prime \prime \prime} \rightarrow X^{\prime}$ be the restriction mapping. Since $\left(\left(x_{i}^{\prime}\right),\left(S e_{i}\right)\right)$ is shrinking, $\left(\left(S e_{i}\right),\left(x_{i}^{\prime}\right)\right)$ is an atomic decomposition for $X^{\prime}$ with respect to $\left(X_{d}\right)^{\prime}$. For any $x^{\prime \prime \prime}$ in $X^{\prime \prime \prime}$, we can apply the reconstruction formula to $x^{\prime}=\rho\left(x^{\prime \prime \prime}\right)$ to deduce that $\sum_{i=1}^{N}\left\langle x^{\prime \prime \prime}, S e_{i}\right\rangle x_{i}^{\prime}$ is convergent. Then $\left(\left(S e_{i}\right),\left(x_{i}^{\prime}\right)\right)$ is boundedly complete.

The analogous proposition for Schauder bases has a dual result: if a Banach space $X$ has a boundedly complete basis $\left(e_{i}\right)$, then $X$ is isomorphic to the dual of a Banach space with shrinking basis (namely, the closed subspace of $X^{\prime}$ spanned by the dual basic sequence $\left(e_{i}^{\prime}\right)$ ). The fact that the biorthogonal sequence $\left(e_{i}^{\prime}\right)$ is always a basic sequence in $X^{\prime}$ is crucial for the proof of this result.

For an atomic decomposition $\left(\left(x_{i}^{\prime}\right),\left(x_{i}\right)\right)$, we do not know if $\left(\left(x_{i}\right),\left(x_{i}^{\prime}\right)\right)$ is in general an atomic decomposition of the closed subspace of $X^{\prime}$ spanned by $\left(x_{i}^{\prime}\right)_{i}$. If this is the case and $\left(\left(x_{i}^{\prime}\right),\left(x_{i}\right)\right)$ is boundedly complete, it is not difficult to see that $X$ is isomorphic to the dual of $Y=\left[\left(x_{i}^{\prime}\right)_{i}\right]$, and that $\left(\left(x_{i}\right),\left(x_{i}^{\prime}\right)\right)$ is a shrinking atomic decomposition of $Y$.

Also, not knowing if $\left(\left(x_{i}\right),\left(x_{i}^{\prime}\right)\right)$ is an atomic decomposition (of the closure of $\left.\left[\left(x_{i}^{\prime}\right)_{i}\right]\right)$ prevents us from giving, with full generality, a characterization of reflexivity in terms of properties of the atomic decomposition of $X$.

We have:

Proposition 2.4. Let $X$ be a Banach space admitting an atomic decomposition $\left(\left(x_{i}^{\prime}\right),\left(x_{i}\right)\right)$ (with respect to some sequence space $\left.Z\right)$. If $\left(\left(x_{i}^{\prime}\right),\left(x_{i}\right)\right)$ is shrinking and boundedly complete, then $X$ is reflexive. 
Proof. Since $\left(\left(x_{i}^{\prime}\right),\left(x_{i}\right)\right)$ is boundedly complete, for each $x^{\prime \prime} \in X^{\prime \prime}$ there exists $x \in X$ such that $x=\sum_{i=1}^{\infty}\left\langle x^{\prime \prime}, x_{i}^{\prime}\right\rangle x_{i}$ is convergent. As the decomposition is shrinking, for every $x^{\prime} \in X^{\prime}$ we have the reconstruction formula

$$
x^{\prime}=\sum_{i=1}^{\infty}\left\langle x^{\prime}, x_{i}\right\rangle x_{i}^{\prime} .
$$

Then

$$
\left\langle x^{\prime}, x\right\rangle=\sum_{i=1}^{\infty}\left\langle x^{\prime \prime}, x_{i}^{\prime}\right\rangle\left\langle x^{\prime}, x_{i}\right\rangle=\left\langle x^{\prime \prime}, x^{\prime}\right\rangle .
$$

Therefore, $x^{\prime \prime}=x \in X$, which shows that $X$ is reflexive.

We do not know if the converse of the above proposition holds in general. However, we do have a characterization of reflexivity analogous to the one for Schauder bases if we impose some unconditionality assumptions on the atomic decomposition. Let us say that an atomic decomposition $\left(\left(x_{i}^{\prime}\right),\left(x_{i}\right)\right)$ is unconditional if for any $x \in X$, its series expansion $\sum_{i=1}^{\infty}\left\langle x_{i}^{\prime}, x\right\rangle x_{i}$ converges unconditionally. Note that this happens, for example, when the atomic decomposition is associated to an operator $S: X_{d} \rightarrow X$ and the canonical basis of $X_{d}$ is unconditional. With this definition, we have:

TheOREM 2.5. Let $X$ be a Banach space and $X_{d}$ be a Schauder sequence space. Let $S: X_{d} \rightarrow X$ be a continuous operator and $\left(x_{i}^{\prime}\right) \subset X^{\prime}$ be a sequence such that $\left(\left(x_{i}^{\prime}\right),\left(S e_{i}\right)\right)$ is an unconditional atomic decomposition for $X$ with respect to $X_{d}$. Then $X$ is reflexive if and only if $\left(\left(x_{i}^{\prime}\right),\left(S e_{i}\right)\right)$ is shrinking and boundedly complete.

Proof. One implication is given by Proposition 2.4, so suppose that $X$ is a reflexive space. Since any atomic decomposition for $X$ is boundedly complete we only have to show that $\left(\left(x_{i}^{\prime}\right),\left(S e_{i}\right)\right)$ is shrinking. By Theorem 1.4, this is equivalent to $\left(\left(S e_{i}\right),\left(x_{i}^{\prime}\right)\right)$ being an atomic decomposition for $X^{\prime}$ with respect to $\left(X_{d}\right)^{\prime}$. Since $\left\langle x^{\prime}, S e_{i}\right\rangle=\left\langle S^{\prime} x^{\prime}, e_{i}\right\rangle$ and $S J=I_{X}$, it only remains to show that the reconstruction formula holds for any $x^{\prime} \in X^{\prime}$, which means that

$$
x^{\prime}=\sum_{i}\left\langle x^{\prime}, S e_{i}\right\rangle x_{i}^{\prime}
$$

holds for all $x^{\prime} \in X^{\prime}$. Moreover, since the series weakly converges to $x^{\prime}$, we only need to show that it is norm convergent.

The Orlicz-Pettis theorem states that weak subseries convergence implies norm subseries convergence. Select an increasing sequence $\left(i_{k}\right)$ of positive integers and check that $\sum\left\langle x^{\prime}, S e_{i_{k}}\right\rangle x_{i_{k}}^{\prime}$ is weakly convergent. Since $X$ is reflexive, it is enough to show that, for any $x \in X$, the sequence

$$
\left(\left\langle\sum_{k=1}^{N}\left\langle x^{\prime}, S e_{i_{k}}\right\rangle x_{i_{k}}^{\prime}, x\right\rangle\right)_{N}=\left(\left\langle x^{\prime}, \sum_{k=1}^{N}\left\langle x_{i_{k}}^{\prime}, x\right\rangle S e_{i_{k}}\right\rangle\right)_{N}
$$


is Cauchy. Since $\sum_{i}\left\langle x_{i}^{\prime}, x\right\rangle S e_{i}$ is unconditionally convergent in $X$, any subseries is convergent and, in particular, $\sum_{k=1}^{N}\left\langle x_{i_{k}}^{\prime}, x\right\rangle S e_{i_{k}}$ converges. As a result, the sequence in (2.1) is a Cauchy sequence.

Acknowledgements. We are grateful to Carlos Cabrelli and Ursula Molter for introducing us to the subject and for their useful suggestions. We also want to thank Verónica Dimant, Santiago Muro, Damián Pinasco and Nacho Zalduendo for helpful conversations. We are also indebted to the referee for very useful comments and suggestions, and for providing us with Example 1.12.

This project was supported in part by PICT 05 17-33042, UBACyT X108 and UBACyT X038. The first author was also partially supported by PICT 0600587 and the second author by UBACyT X863 and PICT 0600897.

\section{References}

[1] J. J. Benedetto and S. D. Li, Subband coding and noise reduction in multiresolution analysis frames, Proc. of SPIE Conf. on Mathematical Imaging, San Diego, 1994.

[2] D. Carando and S. Lassalle, Atomic decompositions for tensor products and polynomial spaces, preprint.

[3] P. Casazza, Approximation properties, in: Handbook of the Geometry of Banach spaces, Vol. I, W. B. Johnson and J. Lindenstrauss (eds.), Elsevier, 2001, 271-316.

[4] P. Casazza, O. Christensen and D. T. Stoeva, Frame expansions in separable Banach spaces, J. Math. Anal. Appl. 307 (2005), 710-723.

[5] P. Casazza, D. G. Han and D. Larson, Frames for Banach spaces, in: The Functional and Harmonic Analysis of Wavelets and Frames (San Antonio, TX, 1999), Contemp. Math. 247, Amer. Math. Soc., Providence, RI, 1999, 149-182.

[6] O. Christensen and Ch. Heil, Perturbations of Banach frames and atomic decompositions, Math. Nachr. 185 (1997), 33-47.

[7] I. Daubechies, Ten Lectures on Wavelets, SIAM, Philadelphia, PA, 1992.

[8] J. Diestel, Sequences and Series in Banach Spaces, Grad. Texts in Math. 92, Springer, New York, 1984.

[9] R. J. Duffin and A. C. Schaeffer, A class of nonharmonic Fourier series, Trans. Amer. Math. Soc. 72 (1952), 341-366.

[10] B. R. Gelbaum and J. Gil de Lamadrid, Bases of tensor products of Banach spaces, Pacific J. Math. 11 (1961), 1281-1286.

[11] B. Grecu and R. Ryan, Schauder bases for symmetric tensor products, Publ. Res. Inst. Math. Sci. 41 (2005), 459-469.

[12] K. Gröchenig, Describing functions: Atomic decompositions versus frames, Monatsh. Math. 112 (1991), 1-42.

[13] R. C. James, Bases and reflexivity of Banach spaces, Ann. of Math. (2) 52 (1950), $518-527$.

[14] -, A non-reflexive Banach space isometric with its second conjugate space, Proc. Nat. Acad. Sci. U.S.A. 37 (1951), 174-177.

[15] J. Lindenstrauss and L. Tzafriri, Classical Banach Spaces I, II, Springer, 1977.

[16] N. J. Munch, Noise reduction in tight Weyl-Heisenberg frames, IEEE Trans. Inform. Theory 38 (1992), 608-616. 
[17] A. Pełczyński, Any separable Banach space with the bounded approximation property is a complemented subspace of a Banach space with a basis, Studia Math. 40 (1971), 239-243.

[18] I. Singer, Bases in Banach Spaces I, Springer, New York, 1970.

Departamento de Matemática - Pab I

Facultad de Cs. Exactas y Naturales

Universidad de Buenos Aires

(1428) Buenos Aires, Argentina

and

CONICET

E-mail: dcarando@dm.uba.ar slassall@dm.uba.ar

Received February 2, 2008

Revised version June 25, 2008 